\title{
Mechanism of the Developed Sensorimotor Therapy Device: Synchronous Inputs of Visual Stimuli and Vibration to Improve Recovery of Distal Radius Fractures
}

\author{
Toyohiro Hamaguchi* and Hiromi Nakamura Thomas* \\ Department of Rehabilitation, Graduate School of Health Sciences, Saitama Prefectural University, Japan
}

*Corresponding author: Toyohiro Hamaguchi and Hiromi Nakamura Thomas, Department of Rehabilitation, Graduate School of Health Sciences, Saitama Prefectural University, Japan, Email: hamaguchi-toyohiro@spu.ac.jp \& nakamura-hiromi@spu.ac.jp

\section{ARTICLE INFO}

Received: 慧 August 06, 2021

Published: August 18, 2021

Citation: Toyohiro Hamaguchi, Hiromi Nakamura Thomas. Mechanism of the Developed Sensorimotor Therapy Device: Synchronous Inputs of Visual Stimuli and Vibration to Improve Recovery of Distal Radius Fractures. Biomed J Sci \& Tech Res 38(2)-2021. BJSTR. MS.ID.006120.

\section{ABSTRACT}

Every fracture requires immobilization following surgery; however, immobilization impairs tactile perception and consequently diminishes cortical somatosensory maps. To minimize this adverse phenomenon, we developed a prototype device (development code: Ghost) for post-operative use of patients who underwent surgery for distal radius fracture (DRF). Ghost consists of a combination of a visualizer paired with a headmounted display to provide visual stimuli for enhance motor imagery and a vibration motor to provide pallesthetic feedback on the extensor carpi ulnaris tendon of the intact side. This study reports on the mechanism and theoretical foundation on which the operation of Ghost is currently built.

\section{Introduction}

Each fracture requires immobilization following surgery. However, this immobilization impairs tactile perception and causes diminishing of cortical somatosensory maps [1]. This adverse event occurs not only among patients with undergoing immobilization for fracture, but also among their healthy counterparts [1,2]. Decreased limb use can lead to changes in the cortical representation of involved muscles [3]. These changes represent a disuse-dependent type of plasticity [4]. Because of the adverse effect arising from immobilization, we observed that some patients with distal radius fractures (DRFs) complained that prior sensation was not restored in the affected limb or that they forgot how to move the affected limb following immobilization phase, postoperatively. These patients are encouraged to further engage these limbs in active motion. Figure 1 illustrates the disuse-dependent plasticity from wrist fixation in the acute phase of patients with DRF.
The process on the left illustrates insufficient coding. When joint movement of an upper limb is restricted for a certain period of time, brain activity is correspondingly reduced. As the illustration on the right shows, reduced brain activity precipitates a disuse-dependent type of plasticity that causes encoding failure, resulting in failed cerebral activation of pathways involved in the target movement or delayed recall of such movement pathways. To minimize such negative consequences of disuse-dependent plasticity during the immobilization phase, and to maintain tactile perception and somatosensory cortical maps, we developed a prototype device (development code: Ghost, Patent No. 6425355) that may be applicable to patients with DRF in the postoperative period. 


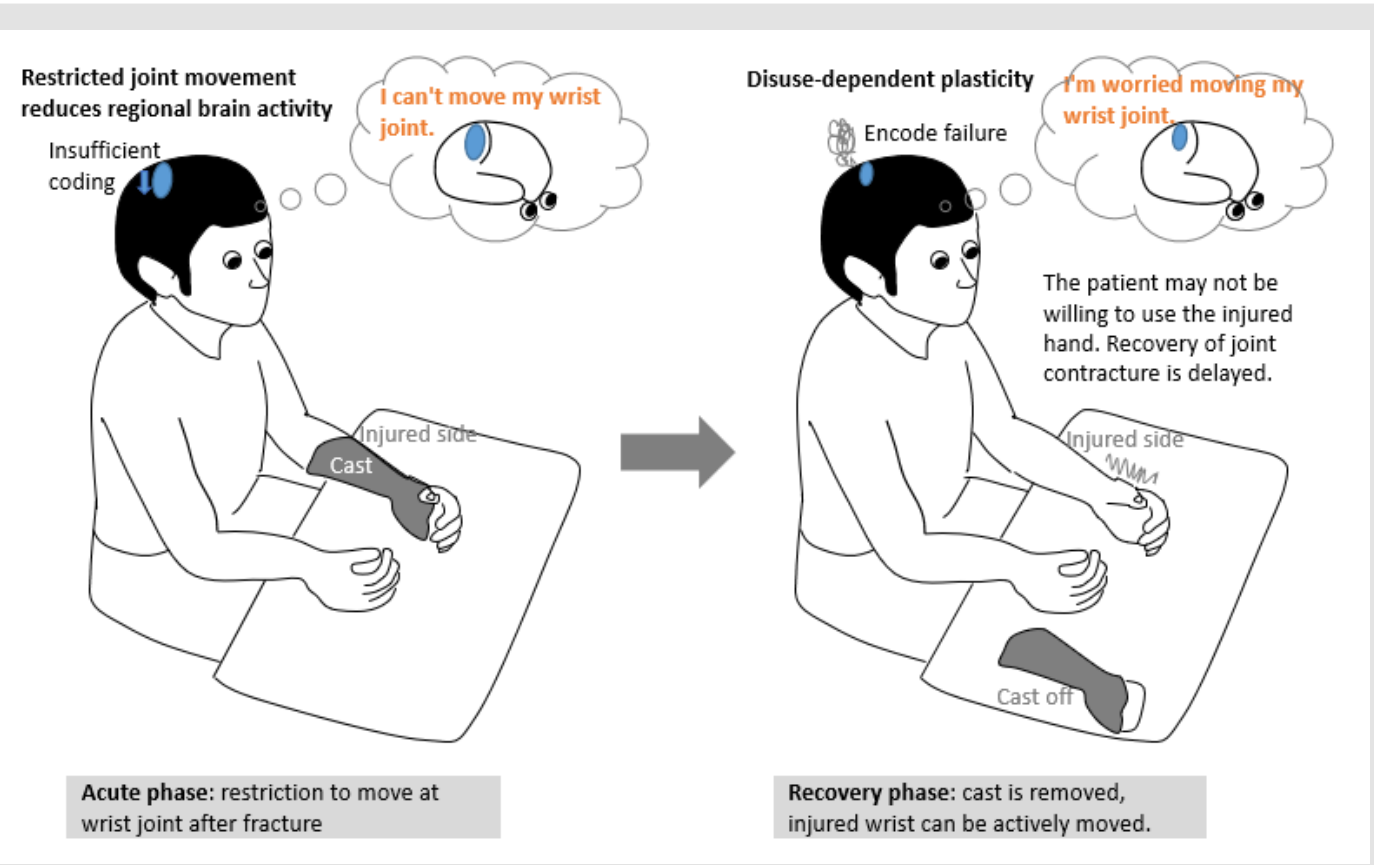

Figure 1: Disuse-dependent plasticity caused by wrist immobilization.

\section{Ghost: Out Prototypical Device}

Ghost was developed to synchronously provide both visual and vibration feedback. Ghost employs a head-mounted VR display for viewing videos and a vibrating motor to elicit pallesthesia.

Figure 2 shows the Ghost system as it synchronously stimulates visualization and vibratory sense among patients with DRF. The visual stimuli are implemented with a visualizer, which is expected to enhance motor imagery of limb motion. The device was built using
(1) A head-mounted display (Oculus virtual reality (VR); Irvine, California, USA),

(2) A control unit (MacBook Pro; Apple Inc., Cupertino, California, USA), and

(3) An originally developed three-dimensional video application. The vibratory stimuli were applied through a vibration motor with an operating frequency of 70 to $80 \mathrm{~Hz}$ (Daito Electric Machine Industry Co., Ltd., Osaka, Japan, 2015).

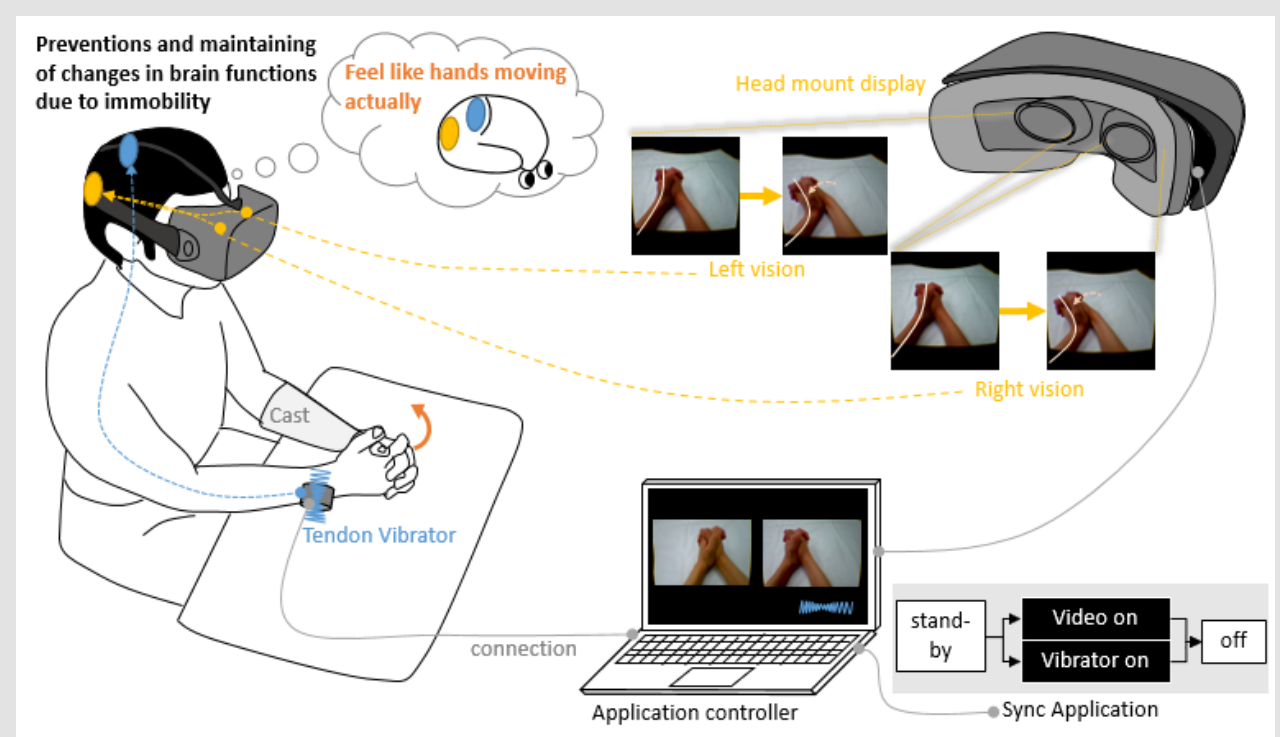

Figure 2: The Ghost system synchronously implements visualization and vibration stimuli to prevent disuse-dependent plasticity in the acute phase for patients with distal radius fracture. 
This vibration motor was placed on the extensor carpi ulnaris tendon on the contralateral, intact side. The vibratory stimulus was accordingly applied concurrently with three-dimensional visualization. The combined stimuli were programmed to last for $30 \mathrm{~s}$, with a 10-s interval allocated for rest. A patient on whom Ghost was tested reported perception of palmar flexion of the wrist upon application of vibratory stimulus with the vibration motor This perception occurred despite the joint not engaging in any motion [5]. Upon repeat testing, concurrent application of vibratory stimulus on both the injured hand and the contralateral healthy hand, yielded a sensation that corresponded with non-dorsiflexion of the fractured hand. [6]. In the head-mounted display, a parallax image of a hand in dorsiflexion and palmar flexion was concurrently shown. Upon visualization of each parallax image, the participants felt ownership of the hand as it moved in a three-dimensional movie. A sense of ownership of one's limb is essential for effective visual stimuli $[7,8]$. In this implementation, Ghost provided concurrent visual and vibration stimuli. When multiple stimuli for different senses are elicited, humans perceive the experience as more "real" than if only a single stimulus were implemented [9].

\section{The Mechanism and the Theoretical Foundations of Ghost}

The novelty of Ghost is in the synergistic effect of visual and vibration stimuli for patients recovering from DRF while in the immobilization phase. We expected that vibration stimuli would amplify the effects of motor imagery over merely providing exclusively visual stimuli, based on previous studies [9]. The combination of vibration stimuli and visual stimuli was examined in healthy participants, showing the importance of carefully selecting specific visual feedback to improve the illusion of movement induced by tendon vibration. Moreover, the illusion was enhanced by adding VR cues congruent with the illusion of movement [10]. Motor imagery practice using VR is used for various patients. It was found to be beneficial for patients with stroke, to improve their activities of daily living [11]. A systematic review and metaanalysis showed that the use of specialized VR and VR gaming can be advantageous for the treatment of the upper extremity; however, this does not have the same effect for hand dexterity and gait, for all pathologies considered [12].

Motor imagery practice is beneficial for healthy people during arm immobilization [13]. Key to the success of practice with motor imagery is whether participants were able to experience ownership [7]. Moreover, vibration stimuli have the potential to enhance illusory hand movement and corresponding activity of the primary motor cortex [14,15]. Using Ghost, its effectiveness was tested among postoperative patients who were treated for DRF. Clinical applications for Ghost are still under the experimental phase. Frenkel, et al. reported that motor imagery declined temporarily when the wrist joint was temporarily fixed [16]. Images should be improved to allow patients with DRF to experience body ownership, such that they would feel as if they were moving their very own limbs. In addition, three-dimensional visual stimuli cause visual fatigue [17]. Therefore, duration of practice must accordingly be considered. Diabetes and peripheral neuropathy often diminish vibratory sensation. Patients with DRF who have such comorbidities do not experience proprioception in the same way as healthy subjects. For patients with DRF and somatosensory impairment, it is worth considering to increase vibration intensity.

\section{Conclusion}

In the immobilization phase for the treatment of DRF, the wrist joint must be immobilized. Consequently, a disuse-dependent plasticity occurs from non-use of the injured hand. Because of such disuse-dependent plasticity, some patients with DRF may not be able to use the affected hand properly for an unspecified period of time following the immobilization phase. If Ghost is used for patients with DRF in the acute phase, the risk of disuse-dependent plasticity may be further reduced, and tactile perception and somatosensory cortical maps may be maintained. Further studies will verify whether this intervention can be effective for patients with DRF. In addition, there are issues that hound its practical application, such as the effective optimization of the combined multisensory stimulations, the individualization of stimulation intensity and frequency for each patient, and the feasibility of utilization for both the acute and convalescent phases.

\section{References}

1. Lissek S, Wilimzig C, Stude P, Pleger B, Kalisch T, et al. (2019) Immobilization impairs tactile perception and shrinks somatosensory cortical maps. Curr Biol 19(10): 837-842.

2. Villatte J, Taconnat L, Bidet Ildei C, Toussaint L (2021) Short-term upper limb immobilization and the embodied view of memory: A pilot study. PLoS One 16(3): e0248239.

3. Ngomo S, Leonard G, Mercier C (2012) Influence of the amount of use on hand motor cortex representation: effects of immobilization and motor training. Neuroscience 220: 208-214.

4. Button DC, Kalmar JM (2019) Understanding exercise-dependent plasticity of motoneurons using intracellular and intramuscular approaches. Appl Physiol Nutr Metab 44(11): 1125-1133.

5. Naito E, Roland PE, Ehrsson HH (2002) I feel my hand moving: a new role of the primary motor cortex in somatic perception of limb movement. Neuron 36(5): 979-988

6. Imai R, Osumi M, Morioka S (2016) Influence of illusory kinesthesia by vibratory tendon stimulation on acute pain after surgery for distal radius fractures: a quasi-randomized controlled study. Clin Rehabil 30(6): 594-603.

7. Kaneko F, Blanchard C, Lebar N, Nazarian B, Kavounoudias A, et al. (2015) Brain Regions Associated to a Kinesthetic Illusion Evoked by Watching a Video of One's Own Moving Hand. PLoS One 10(8): e0131970.

8. Van der Stoep N, Nijboer TC, Van der Stigchel S, Spence C (2015) Multisensory interactions in the depth plane in front and rear space: a review. Neuropsychologia 70: 335-349. 
9. Blanke O, Slater M, Serino A (2015) Behavioral, Neural, and Computational Principles of Bodily Self-Consciousness. Neuron 88(1): 145-166.

10. Le Franc S, Fleury M, Cogne M, Butet S, Lecuyer A, et al. (2020) Influence of virtual reality visual feedback on the illusion of movement induced by tendon vibration of wrist in healthy participants. PLoS One 15(11): e0242416.

11. Faria AL, Andrade A, Soares L, SB IB (2016) Benefits of virtual reality based cognitive rehabilitation through simulated activities of daily living: a randomized controlled trial with stroke patients. J Neuroeng Rehabil 13(1): 96.

12. Rutkowski S, Kiper P, Cacciante L, Cieślik B, Mazurek J, et al. (2020) Use of virtual reality-based training in different fields of rehabilitation: A systematic review and meta-analysis. J Rehabil Med 52(11): jrm00121.

\section{ISSN: 2574-1241}

DOI: 10.26717/BJSTR.2021.38.006120

T Hamaguchi, H Nakamura Thomas. Biomed J Sci \& Tech Res

(c) (P) This work is licensed under Creative

Submission Link: https://biomedres.us/submit-manuscript.php
13. Debarnot U, Perrault AA, Sterpenich V, Legendre G, Huber C, et al. (2021) Motor imagery practice benefits during arm immobilization. Sci Rep 11(1): 8928.

14. Kito T, Hashimoto T, Yoneda T, Katamoto S, Naito E (2006) Sensory processing during kinesthetic aftereffect following illusory hand movement elicited by tendon vibration. Brain Res 1114(1): 75-84.

15. Naito E (2004) Sensing limb movements in the motor cortex: how humans sense limb movement. Neuroscientist 10(1): 73-82.

16. Frenkel MO, Herzig DS, Gebhard F, Mayer J, Becker C, et al. (2014) Mental practice maintains range of motion despite forearm immobilization: a pilot study in healthy persons. J Rehabil Med 46(3): 225-232.

17. Chen C, Wang J, Li K, Liu Y, Chen X (2015) Visual fatigue caused by watching 3DTV: an fMRI study. Biomed Eng Online 14(1): S12.

$\begin{array}{ll}\text { BIOMEDICAL } & \text { Assets of Publishing with us } \\ \text { RESEARCHES } & \text { - Global archiving of articles } \\ \text { - Immediate, unrestricted online access }\end{array}$

